\title{
Micelle formation and red-light fluorescence emission of benzothiadiazole-triphenylamine amphiphilic molecules in water/methanol solutions explored with synchrotron small-angle X-ray scattering
}

\author{
Shimpei Yamada ${ }^{1}$, Yusuke Sanada ${ }^{1}$, Shota Fujii ${ }^{1}$, Ikumi Kitahara ${ }^{2}$, Isamu Akiba ${ }^{1}$, Tsutomu Ishi-I ${ }^{2}$ \\ and Kazuo Sakurai ${ }^{1}$
}

Polymer Journal (2016) 48, 973-976; doi:10.1038/pj.2016.56; published online 8 June 2016

\section{INTRODUCTION}

Fluorescent organic dyes that emit red and infrared light, ranging from 600 to $1000 \mathrm{~nm}$ in wavelength, have quite a wide range of applications as biomarkers because light of these wavelengths is highly transmissible through organs and cells. One way to create such red-light fluorescence is to use the interaction between electron-donor and electron-acceptor moieties, which in most cases are covalently bonded nearby each other. ${ }^{1}$ Benzothiadiazole is a strong electronaccepting moiety because the thiadiazole withdraws electrons from the adjacent aromatic ring and has the ability to strongly fluoresce. ${ }^{2}$ Recently, Ishi-i et al. ${ }^{3,4}$ reported that when benzothiadiazole is combined with electron-donating triphenylamines, an intense red light is emitted owing to the electron donor-acceptor interaction. Interestingly, the intermolecular donor-acceptor conjugates do not suffer from concentration quenching; thus, even aggregates or crystals of these conjugates fluoresce.

The fluorescence intensity is mainly determined by competition between the lifetime of the excited state and the rate at which the excited state is deactivated by non-radiative processes. However, the luminous efficiency of longer-wavelength emissions decreases in polar aqueous media because the highly polarized excited state arising from the donor-acceptor interaction increases the formation of a nonradiative deactivation channel. In other words, the emission of the donor-acceptor system is sensitive to the surrounding environment such as the polarity and the hydrogen bonding ability of a solvent. ${ }^{5-10}$ In a polar aqueous environment, the donor-acceptor dye tends to adopt a highly polarized excited state such as the charge transfer state, ${ }^{11,12}$ from which the formation of the non-radiative deactivation channel is accelerated, leading to strong fluorescence quenching. Therefore, in polar solutions, the emission is quenched, which reduces fluorescence efficiency. The extent of fluorescence quenching correlates with the increase in polarity, and this process facilitates the transition from a locally excited state to a highly polarized excited state such as an intramolecular charge transfer state. The polarized excited state is stabilized by the solvation provided by polar solvent molecules that increases the non-radiative deactivation.

Indeed, the red-light emitting donor-acceptor molecules never fluoresce in aqueous solutions. This solvent-induced quenching is a serious drawback when such molecules are applied in a biological system. Ishi-i et al. ${ }^{13}$ found that solvent-induced quenching can be prevented by the aggregation of dye molecules. They measured the fluorescence intensity of a benzothiadiazole/triphenylamine conjugate as a function of the water content in THF/water mixtures. In tetrahydrofuran (THF), the molecules were found to be dispersed and emitted a strong red light. With increasing water content, the emission intensity dramatically decreased and reached a minimum at $60 \mathrm{vol} \%$ of water. Interestingly, the further addition of water induced a recovery of the emission. In accordance with these fluorescence changes, dynamic light scattering showed the appearance of aggregates, and the absorption maximum shifted bathochromically, indicating that J-type stacking had occurred. ${ }^{14}$ The authors concluded that the hydrophobicity of both the benzothiadiazole and triphenylamine moieties induced an aggregate in water and excluded water molecules from the vicinity of the dye molecules. They named this phenomenon 'aggregate-induced emission'. ${ }^{15}$ More recently, Ishi-i et al. ${ }^{3}$ attached a hydrophilic moiety to the benzothiadiazole/triphenylamine conjugate (Figure 1) to make the conjugate amphiphilic. The authors reported that the resultant molecules (denoted benzothiadiazole-triphenylamine (BT)-lipid) formed a stable micelle in aqueous solutions and emitted a strong red light. It seems that this stable aggregate formation due to micellization is an excellent method for eliminating secondary aggregation and precipitation. Following on from the previous study, ${ }^{3}$ we explored BT-lipid micelle structures in methanol/water mixtures by use of synchrotron small-angle X-ray scattering (SAXS). One of the 
main aims was to determine the aggregation number at which red light starts to be emitted and other criteria for this phenomenon.

\section{EXPERIMENTS}

Synthesis and sample preparation for synchrotron small-angle X-ray scattering

BT-lipid, that is, 4-[4-(N,N-diphenylamino)phenyl]-7-[3,5-bis (3,6,9,12,15,18-hexaoxanonadecyloxy)phenyl]-2,1,3-benzothiadiazole (Figure 1), was synthesized according to the reported procedure. ${ }^{3}$ To a solution of 2-[2-(2-\{2-[2-(2-methoxy-ethoxy)-ethoxy]-ethoxy\}ethoxy)-ethoxy]-ethyl p-tosylate in dry dimethylformamide were added 4-(3,5-dihydroxyphenyl)-7-[4-(N,N-diphenylamino)phenyl]2,1,3-benzothiadiazole and crushed potassium carbonate $(276 \mathrm{mg}$, $2.0 \mathrm{mmol}$ ) under an argon atmosphere. The resultant mixture was heated and acidified, which was followed by extraction with ethyl acetate and purification by the described method. ${ }^{3}$

\section{Synchrotron small-angle X-ray scattering}

All the SAXS measurements were performed at beam line 40B2 of the SPring-8 facility (Hyogo, Japan). The sample solution was loaded into

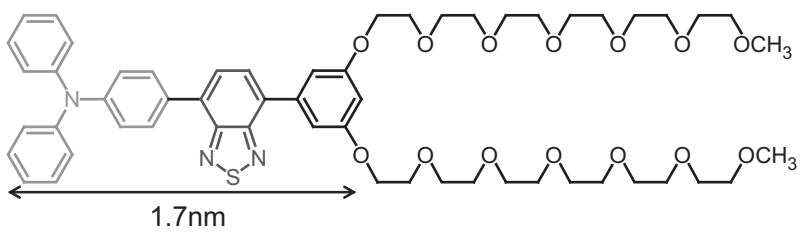

Figure 1 The chemical structure of the amphiphilic benzothiadiazole - triphenylamine molecule (BT-lipid) studied in this paper. Red and blue indicate the electron donor and acceptor, respectively. A full color version of this figure is available at Polymer Journal online. a quartz capillary cell (Hilgenberg GmbH, Malsfeld, Germany). The cell was then mechanically sealed with a silicone rubber gasket and placed in a vacuum chamber. The X-ray wavelength $(\lambda)$ and the sample-to-detector distance were $0.10 \mathrm{~nm}$ and $1.0 \mathrm{~m}$. The scattered $\mathrm{X}$-rays were detected with a $30 \mathrm{~cm} \times 30 \mathrm{~cm}$ imaging plate (RAXIS VII, Rigaku, Tokyo, Japan). After making the appropriate transmittance correction, the degree of solvent scattering was subtracted from that of the solution, yielding the SAXS profiles of the solutes. The concentrations of the BT-lipid samples were $1.0 \mathrm{mg} \mathrm{ml}^{-1}$. We confirmed that the particle interference was negligibly small at these concentrations (Supplementary Information, Supplementary Figure S1).

\section{Fluorescence quantum yield}

The quantum yield was measured using an absolute photoluminescence quantum yield measurement system (C9920-02; Hamamatsu, Shizuoka, Japan). This instrument consisted of an integrating sphere equipped with a monochromatized Xe arc lamp as the light source and a multichannel spectrometer. The sensitivity of this system was fully calibrated for the spectral region 250-950 nm using deuterium and halogen standard light sources. A $10 \mathrm{~mm}$ path length quartz cuvette for the solution samples was set in the integrating sphere.

\section{RESULTS AND DISCUSSION}

Figure 2 shows how the SAXS profiles change as the water/methanol composition (water vol\%: wf) was changed, as well as the fluorescence color. As Ishi-i et al. ${ }^{3}$ reported, BT-lipids dissolve completely in methanol; thus, no fluorescence was observed, because of solventinduced non-radiative deactivation. Although no fluorescence was emitted for values of $w f<60 \%$, the SAXS profile began to change at $\mathrm{wf}=30 \%$, indicating that BT-lipids formed an aggregate at a much lower wf than that at which they started to emit fluorescence. With this composition $(\mathrm{wf}=30 \%)$, the scattering intensity monotonically a

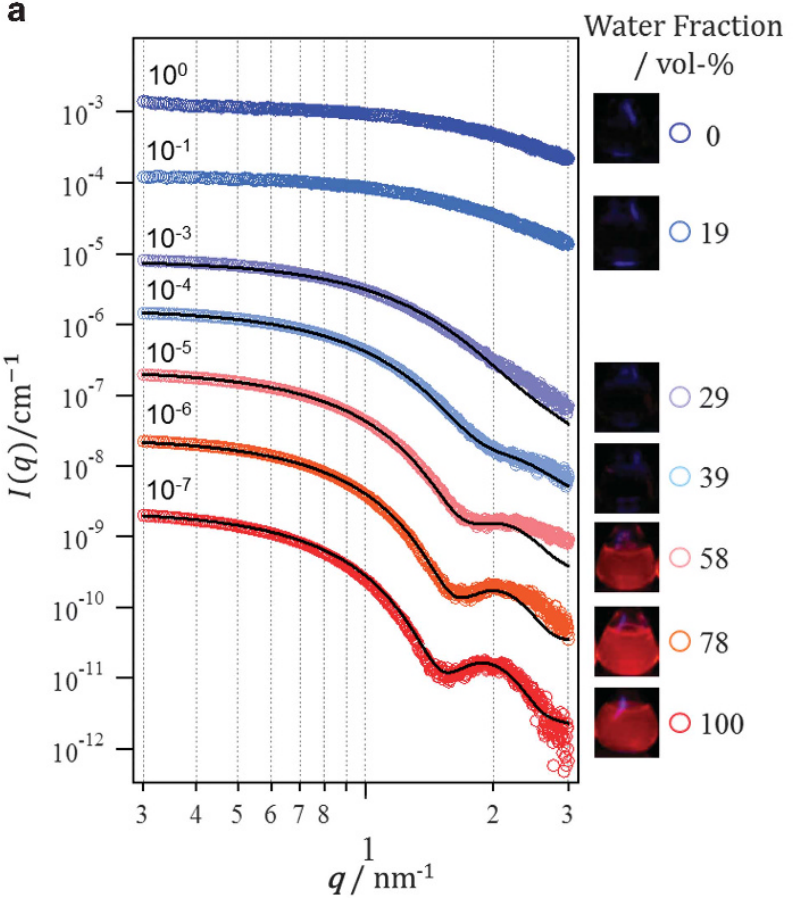

b

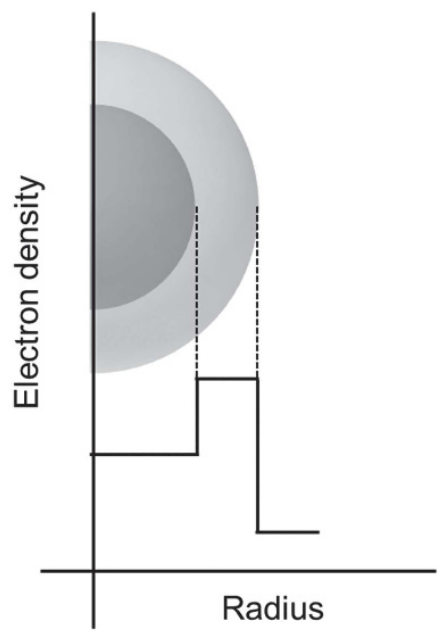

Figure 2 (a) The dependence of SAXS profiles of BT-lipids on the water composition (wf), and the corresponding fluorescence emission images. The SAXS measurements were performed at BL-40B2 of SPring-8, and the absolute X-ray scattering intensity was determined using the absolute scattering intensity of water. The profiles were vertically shifted for convenience by multiplying by the values indicated above each curve. The solid lines that are overlaid on each profile are the best fitting curves calculated from Equation 1. (b) A schematic illustration of the core-shell model. 
Table 1 Summary of the fitting parameters that give the best fitting curves in Figure 2a

\begin{tabular}{lcccccc}
\hline wf/vol\% & $R_{\mathrm{C}}(\mathrm{nm})$ & $R_{\mathrm{S}}(\mathrm{nm})$ & $\rho_{\mathrm{C}}{ }^{\mathrm{a}}$ & $\rho_{\mathrm{S}}{ }^{\mathrm{a}}$ & $\rho_{0}{ }^{\mathrm{a}}$ & $\sigma_{\mathrm{C}} \mathrm{R}_{\mathrm{C}}{ }^{-1}$ \\
\hline 0 & - & - & - & - & 386 & \\
19 & - & - & - & - & 387 & \\
29 & 1.70 & 1.93 & 379 & 382 & 384 & 0.220 \\
39 & 2.00 & 2.14 & 375 & 369 & 380 & 0.220 \\
58 & 2.29 & 2.43 & 362 & 353 & 367 & 0.150 \\
78 & 2.36 & 2.60 & 356 & 361 & 350 & 0.125 \\
100 & 2.38 & 2.90 & 339 & 340 & 334 & 0.110 \\
\hline
\end{tabular}

aElectron densities (electron $\mathrm{nm}^{-3}$ ).

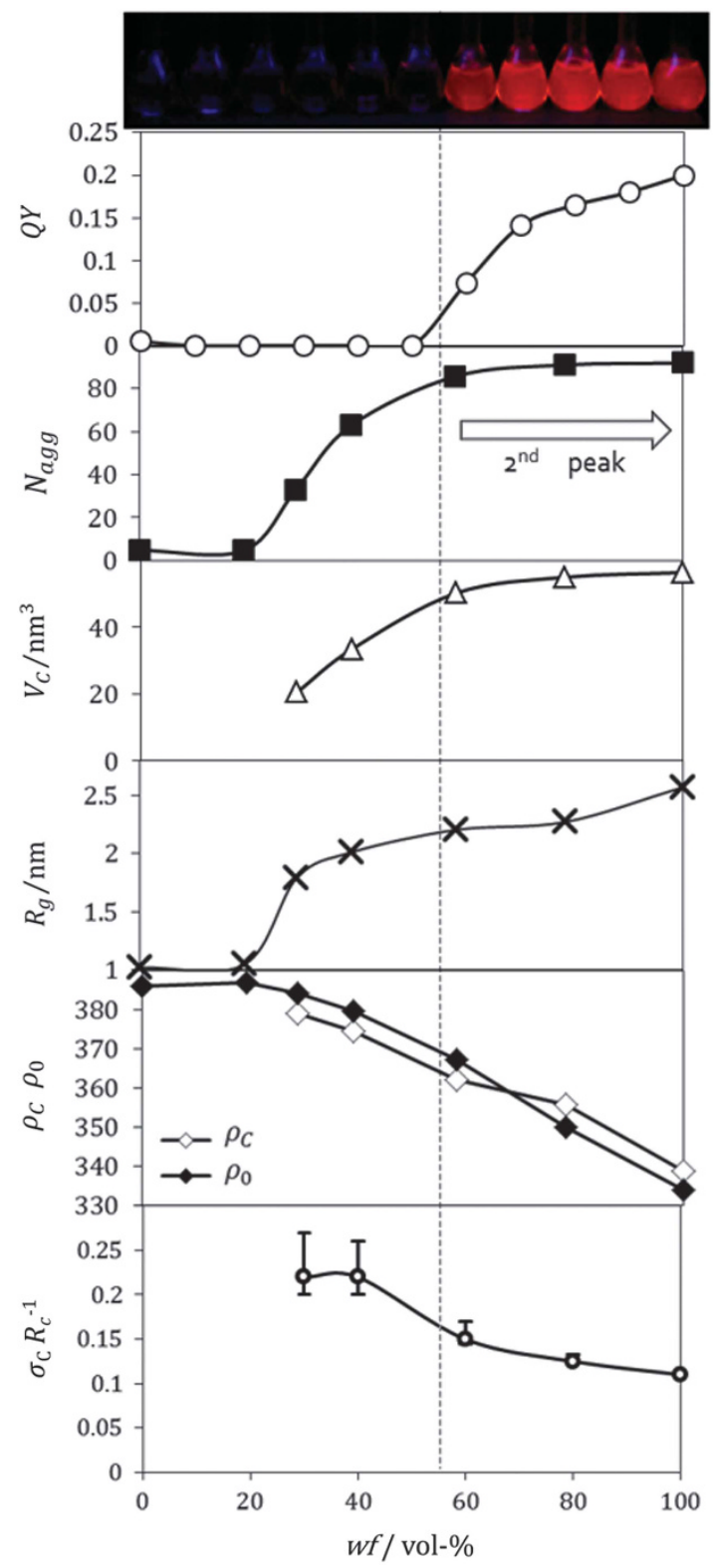

Figure 3 The dependence of the fluorescence and the micellar structure parameters on the water composition. The lowest plot shows how many water molecules bind to each lipid. decreased with an increase in $q$, indicating that the size and shape distributions were quite large and that there might be no clear boundary between the solvent and the micelle. In other words, the aggregate had not grown enough to be called a micelle in terms of SAXS. There was a clear second maximum observed in the SAXS profiles at $\mathrm{wf}=60 \%$. At $\mathrm{wf}>60 \%$, the second maximum was clearer and shifted to lower values of $q$. The profiles for wf $>60 \%$ exhibited a typical feature of spherical core-shell micelles. All of the samples showed a Guinier region; thus, we could determine the aggregation number, $N_{\text {agg }}$.

The scattering profiles for wf $>60 \%$ show typical features of spherical micelles; thus, we presumed that the scattering profile could be fitted by the following equation for a core-shell spherical model. Here, the core-shell model for SAXS analysis indicates an electron density profile that represents core-shell spherical micelles as two concentric spheres with different radii and electron densities (see Figure $2 \mathrm{~b}$ for the schematic illustration).

$$
\begin{aligned}
I(q) & =N\left\{\left(\rho_{c}-\rho_{s}\right) V_{c} \frac{3\left[\sin \left(q R_{c}\right)-q R_{c} \cos \left(q R_{c}\right)\right]}{\left(q R_{c}\right)^{3}}\right. \\
& \left.+\left(\rho_{s}-\rho_{0}\right) V_{s} \frac{3\left[\sin \left(q R_{s}\right)-q R_{s} \cos \left(q R_{s}\right)\right]}{\left(q R_{s}\right)^{3}}\right\}^{2}
\end{aligned}
$$

Here, $q$ is the magnitude of the scattering vector defined by $q=\frac{4 \pi}{2} \sin \left(\frac{\theta}{2}\right)$, where $\lambda$ and $\theta$ are the wavelength of the X-rays and the scattering angle, respectively. $N$ is the number of the micelles in a unit volume, and $\rho_{C}, \rho_{S}$ and $\rho_{0}$ are the electron densities of the core, shell and solvent, respectively. $R_{C}$ and $R_{S}$ are the radii of the core and micelle, respectively, as depicted in Figure 2, and $V_{C}$ and $V_{S}$ are their volumes, respectively. We introduced a Gaussian distribution with a s.d. of $\sigma_{C}$. For fitting the data, $\rho_{0}$ was calculated from the density of the water/methanol mixture by using $\rho_{0 \text {,water }}=334 \mathrm{enm}^{-3}$ and $\rho_{0 \text {,methanol }}=386 \mathrm{enm}^{-3} \cdot{ }^{16}$ Furthermore, we assumed a Gaussian distribution in the core and shell radii. ${ }^{16}$ At the lower $q$ limit, Equation 1 can be written as

$$
\begin{aligned}
& \ln I(q)=\ln I(q \rightarrow 0)-\frac{1}{3}\left(R_{g} q\right)^{2}, \\
& \frac{I(q \rightarrow 0)}{c}=\frac{M_{w}}{N_{A}} v^{2}(\Delta \rho)^{2}
\end{aligned}
$$

Here, $R_{g}, M_{w}, N_{A}, \nu$ and $\Delta \rho$ are the radius of gyration, the weightaveraged molecular weight of one micelle, the Avogadro number, the specific volume and the averaged electron density difference between the solvent and the micelle. After extrapolating the intensity to the value at $q=0$, that is, $I(q \rightarrow 0)$ by using the Guinier plot (Supplementary Information, Supplementary Figure S2), we obtained $R_{g}$ and $M_{w}$; thus, the aggregation number, $N_{a g g}$, could be obtained. Table 1 summarizes the obtained parameters, and the calculated best fit curves (solid lines in Figure 1) are compared with the measured data. The agreement between the data and the calculated values is quite good, so we conclude that BT-lipids indeed form a special coreshell shape.

Figure 3 shows the dependence on wf of quantum yield, $N_{a g g}, V_{C}$, $R_{g}, \rho_{C}, \rho_{0}$ and $\sigma_{C}$. As shown in Figure 2a, the micellar formation began at a much lower wf than that at which fluorescence appeared. At $\mathrm{wf}=30$ and $40 \%$, where there was no emission, the micelles had a lower $N_{a g g}$ and the size distribution was larger than that for $\mathrm{wf} \geqslant 60 \%$. The larger distribution at $\mathrm{wf}=30 \%$ explains why no second intensity maximum was observed for this composition. At $\mathrm{wf}=80 \%, N_{a g g}$ and $V_{C}$ were nearly the same values as at $\mathrm{wf}=100 \%$, 


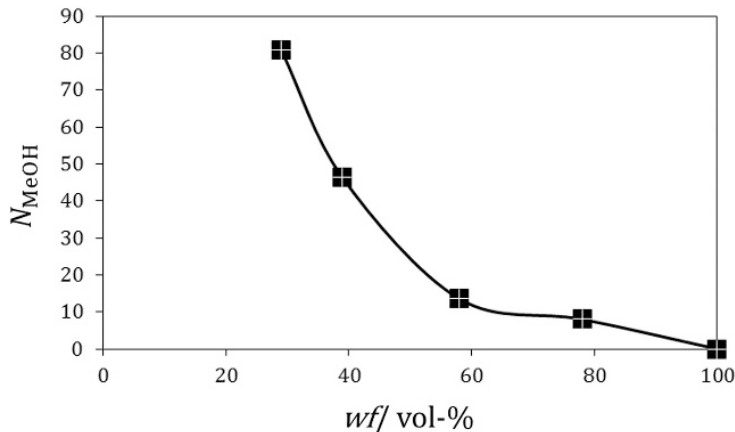

Figure 4 The dependence of the number of bound methanol molecules in the core per BT-lipid on the water composition.

whereas $R_{g}$ was higher and $\rho_{C}$ was lower at wf $=100 \%$. These findings indicated that the micellar structure was still changing or growing in this region upon the addition of water, as the aggregation number was approximately the same. Because methanol is a good solvent for BTlipids and can dissolve the benzothiadiazole/triphenylamine conjugate, we presume that there is some methanol in the core at $\mathrm{wf}=60$ and $80 \%$. The presence of bound methanol (a quencher) would explain why quantum yield is still increasing in this region. Because $\rho_{0}$, methanol $>\rho_{0 \text {,water }}$ and $\rho_{0 \text {,methanol }}>\rho_{C}$ in water, the decrease in $\rho_{C}$ with the increase in wf is related to the amount of core-bound methanol.

We estimated how many methanol molecules $\left(\mathrm{N}_{\mathrm{MeOH}}\right)$ bind to one BT-lipid, assuming the following relation:

$$
\rho_{C}(\mathrm{wf})=\varphi \rho_{0, \text { methanol }}+(1-\varphi) \rho_{C, \mathrm{wf}=100}
$$

Figure 4 plots $N_{\mathrm{MeOH}}$ against wf. From this analysis, we conclude that there is a critical $N_{\mathrm{MeOH}}$ for completely quenching the studied benzothiadiazole/triphenylamine conjugate, and this value is approximately $N_{\mathrm{MeOH}}=10$. The extent to which $N_{\mathrm{MeOH}}$ can be decreased depends on wf, and the exclusion of methanol molecules from the core reduces quenching.

\section{CONCLUSION}

To summarize the present work, we carried out SAXS measurements on BT-lipids in water/methanol mixtures and compared the fluorescence emissions. BT-lipids began to form an aggregate at a much lower wf than that at which they emitted fluorescence. At this composition ( $\mathrm{wf}=60 \%$ ), there was no second maximum observed in SAXS, indicating immature micelle formation. With an increase in wf, the micelles grew and started to show a second maximum in SAXS profiles, which is a typical feature of core-shell micelles. $N_{\text {agg }}$ was evaluated from the Guinier analysis, and it reached approximately 80 at wf $>60 \%$. By fitting the data with a core-shell model, $V_{C}$ and $\rho_{C}$ were determined. From these, $N_{\mathrm{MeOH}}$ was estimated. At the critical wf where emission occurred, $\sim 10$ methanol molecules were present per BT-lipid.

\section{CONFLICT OF INTEREST}

The authors declare no conflict of interest.

\section{ACKNOWLEDGEMENTS}

All SAXS measurements were carried out at SPring-8 BL-40B2 (2014A1268, 2014A1268, 2013B1203, 2013A1564, 2013A1594 and 2013A1207).

1 Chen, C.-T. Evolution of red organic light-emitting diodes: materials and devices. Chem. Mater. 16, 4389-4400 (2004).

2 Raimundo, J.-M., Blanchard, P., Brisset, H., Akoudad, S. \& Roncali, J. Proquinoid acceptors as building blocks for the design of efficient $\pi$-conjugated fluorophores with high electron affinity. Chem. Commun. 11, 939-940 (2000).

3 Ishi-i, T., Kitahara, I., Yamada, S., Sanada, Y., Sakurai, K., Tanaka, A., Hasebe, N., Yoshihara, T. \& Tobita, S. Amphiphilic benzothiadiazole-triphenylamine-based aggregates that emit red light in water. Org. Biomol. Chem. 13, 1818-1828 (2015).

4 Ishi-i, T., Ikeda, K., Ogawa, M. \& Kusakaki, Y. Light-emitting properties of donoracceptor and donor-acceptor-donor dyes in solution, solid, and aggregated states: structure-property relationship of emission behavior. RSC Adv. 5, 89171-89187 (2015).

5 Reichardt, C. Solvatochromic dyes as solvent polarity indicators. Chem. Rev. 94, 2319-2358 (1994)

6 Marini, A., Munoz-Losa, A., Biancardi, A. \& Mennucci, B. What is solvatochromism? J. Phys. Chem. B 114, 17128-17135 (2010).

7 Do, J., Huh, J. \& Kim, E. Solvatochromic fluorescence of piperazine-modified bipyridazines for an organic solvent-sensitive film. Langmuir 25, 9405-9412 (2009).

8 Bohne, C., Ihmels, H., Waidelich, M. \& Yihwa, C. N-acylureido functionality as acceptor substituent in solvatochromic fluorescence probes: detection of carboxylic acids, alcohols, and fluoride ions. J. Am. Chem. Soc. 127, 17158-17159 (2005).

9 Lewis, F. D. \& Yang, J.-S. The excited state behavior of aminostilbenes. A new example of the meta effect. J. Am. Chem. Soc. 119, 3834-3835 (1997).

10 Suzuki, A., Nemoto, N., Saito, I. \& Saito, Y. Design of an environmentally sensitive fluorescent 8-aza-7-deaza-2'-deoxyadenosine derivative with dual fluorescence for the specific detection of thymine. Org. Biomol. Chem. 12, 660-666 (2014).

11 Gao, B.-R., Wang, H.-Y., Hao, Y.-W., Fu, L.-M., Fang, H.-H., Jiang, Y., Wang, L., Chen, Q.-D., Xia, H. \& Pan, L.-Y. Time-resolved fluorescence study of aggregation-induced emission enhancement by restriction of intramolecular charge transfer state. J. Phys. Chem. B 114, 128-134 (2009).

12 Gao, B.-R., Wang, H.-Y., Yang, Z.-Y., Wang, H., Wang, L., Jiang, Y., Hao, Y.-W., Chen, Q.-D., Li, Y.-P. \& Ma, Y.-G. Comparative time-resolved study of two aggregation-induced emissive molecules. J. Phys. Chem.C 115, 16150-16154 (2011).

13 Ishi-i, T., Hashimoto, R. \& Ogawa, M. Aggregation of naphthobisthiadiazole-based donor-acceptor-donor dyes that restrict quenching in solution and emit red light in polar aqueous media. Asian J. Org. Chem. 3, 1074-1082 (2014).

14 Kasha, M., Rawls, H. R. \& Ashraf El-Bayoumi, M. The exciton model in molecular spectroscopy. Pure Appl. Chem. 11, 371-391 (1965).

15 Mei, J., Leung, N. L., Kwok, R. T., Lam, J. W. \& Tang, B. Z. Aggregationinduced emission: together we shine, united we soar! Chem. Rev. 115, 11718-11940 (2015).

16 Akiba, I., Terada, N., Hashida, S., Sakurai, K., Sato, T., Shiraishi, K., Yokoyama, M., Masunaga, H., Ogawa, H., Ito, K. \& Yagi, N. Encapsulation of a hydrophobic drug into a polymer-micelle core explored with synchrotron SAXS. Langmuir 26 , 7544-7551 (2010).

Supplementary Information accompanies the paper on Polymer Journal website (http://www.nature.com/pj) 